\title{
Die Adrenalinmydriasis bei chronischer Nephritis.
}

\author{
Von \\ Toyojiro Kato und Masao Watanabe. \\ (加藤豐治 郎) \\ (渡 㭡 正 雄) \\ (Aus Prof. Kato's medizinischer Klinik an der Tohohu \\ Universität zu Sendai.)
}

Bekanntlich lommt die Mydriasisreaktion der Pupille anf Einträufelung von Adrenalin, welche zuerst Loewi ${ }^{1)}$ bei Pankreasdiabetes beobachtet hat, auch bei Basedow'scher Krankheit, sowie bei Erkrankungen von Peritoneum, Bauchorganen, Meningen und Gehirn ${ }^{23 ; 4}$, vor. Kürzlich haben wir an der Hand eines grossen klinischen Materials festgestellt, dass dieses Pupillenphänomen nicht nur bei den oben erwähnten Krankheiten, sondern auch öfters bei Arteriosklerose, Beriberi, zuweilen bei Asthma bronchiale, paroxysmaler Hämoglobinurie, Schwangerschaft, verschiedenen Rückeumarksleiden u. a. provoziert wird, insbesondere aber bei chronischer Nephritis resp. Schrumpfniere ausnahmslos positiv ausfällt.

Ausführung der Reaktion: Man träufelt in den Konjunktivalsack eines Auges zweimal je 4 Tropfen von 1\% Adrenalinlösung (Parke-Davis) im Intervall von 5 Minuten ein, indem man dafür Sorge trägt, dass das Adrenalin möglichst nicht aus dem Konjunktivalsack herıusläuft, und untersucht nach Ablauf einer gewissen Zeit die Differenz der Pupillengrösse beider Seiten. Natürlich muss der Lichteinfall in beide Augen gleichmässig, ferner die Bindeund Hornhaut vollständig intakt, frei von Verletzung bzw. Entzündung sein.

Die Intensität dieser Adrenalinmydriasis bei chronischen Nephritikern ist eine sehr verschiedene. Falls die Reaktion nur schwach ausfällt, bleibt die Differenz der Pupillengrösse ganz minimal, aber in den stark positiven Fällen erreicht sie manchmal mehr als $3 \mathrm{~mm}$ 
im Diameter. Gewöhnlich beginnt sich die Pupille frühestenfalls 5 bis 6 Minuten nach der Einträufelung von Adrenalin, spätestens innerhalb von 15 Minuten $\mathrm{zu}$ erweitern, erreicht den Höhepunkt zwischen 20 bis 60 Minuten und kommt bei schwach positivem Ausfall der Reatition in 1 bis 2 Stunden zur Anfangsgrösse zurück, während sie oft nach 30 Stunden noch Spuren von Mydriasis zeigt, wenn sie auf die Instillation stark reagiert hat; im Durchschnitt dauert die Pupillenerweiterung mehrere Stunden lang. Träufelt man das Adrenalin zu anderer Zeit in das andere Auge ein, reagiert dies nicht immer mit gleich starker Mydriasis.

Diese Pupillenreaktion bei chronischer Nephritis scheint nicht von der Intensität der Albuminurie, sondern von der Höhe des Blutdrucks abhängig zu sein. Hierzu vergleiche man die folgende Tabelle :

Die Beziehung zwischen der Adrenalinmydriasis und dem Blutdruck bei chronischer Nephritis.

\begin{tabular}{l|c|c|c|c|c}
\hline \multirow{2}{*}{$\begin{array}{c}\text { Blutdruck } \\
\text { (R. R.) }\end{array}$} & \multicolumn{2}{|c|}{$\begin{array}{c}\text { Zahl der untersuchten } \\
\text { Fälle }\end{array}$} & \multicolumn{2}{|c}{ unter den positiven Füllen } \\
\cline { 2 - 6 } & negativ & positiv & $\begin{array}{c}\text { schwach } \\
\text { positiv }\end{array}$ & $\begin{array}{c}\text { mittelstark } \\
\text { positiv }\end{array}$ & stark positiv \\
\hline unter $120 \mathrm{~mm}$ & 1 & 7 & $5(71 \%)$ & $2(29 \%)$ & $0(0 \%)$ \\
$120-180 \mathrm{~mm}$ & 1 & 31 & $17(55 \%)$ & $5(16 \%)$ & $9(29 \%)$ \\
über $180 \mathrm{~mm}$ & 0 & 18 & $5(28 \%)$ & $5(28 \%)$ & $8(44 \%)$ \\
& & & &
\end{tabular}

Bei akuter Nephritis kommt die Adrenalinmydriasis nur ausnahmsweise vor. Unter 12 untersuchten Fällen von akuter Nephritis haben nur zwei ganz minimale Pupillenerweiterung auf Adrenalininstillation gezeigt.

Es ist schon früher von Raziejewski5) und Boruttau ${ }^{6}$ darauf hingewiesen, dass bei normalen Menschen Adrenalinmydriasis nie vorkommt. Im Hinblick darauf haben wir bei 36 gesunden Menschen Adrenalininstillation durchgeführt und nur bei zweien eine Spur von Mydriasis bemerkt.

Ungemein schwierig ist es, bei Katzen experimentell chronische Nephritis zu erzeugen; die meisten Tiere gehen bald nach der Einverleibung des Giftes durch akute Nierenschädigung zu Grunde. Uns gelang es nur bei einer Katze mittelst wiederholter Injektion einer minimalen Dose von Uran eine mehrere Monate lang dauernde 
Albuminurie zu provozieren und bei ihr durch Einträufelung von Adrenalin eine sehr starke Mydriasis zu erzeugen. Viele andere Katzen, bei welchen sich durch Injektion von Uran, Sublimat, Arsenik, Chrom u.a. akute Nephritis entwickelte, wiesen keine Adrenalinmydriasis auf.

Aller Wahrscheinlichkeit nach ist die Grundlage der Pupillenreaktion auf Adrenalininstillation keine einheitliche. Ihr positiver Ausfall bei Basedow'scher Krankheit und bei Pankreasdiabetes ist mit einem chemischen Mechanismus verbunden, den man in der veränderten Selkretion von endocrinen Organen erblickt, indem bei der ersteren die Hyperfunktion der Schilddrüse und bei dem letzteren der Ausfall der inneren Sekretion des Pankreas eine gesteigerte Funktion des chromaffinen Systems zur Folge hat. Die von Eppinger, Falta und Rudinger bei normalen sowie bei thyreoidektomierten Hunden experimentell durch fortgesetzte Verfütterung von Schilddrüsensubstanzen erzeugte Adrenalinmydriasis hat analogen Mechanismus. Die positive Pupillenreaktion bei Krankheiten von Peritoneum und Banchorganen führt $\mathrm{Zak}^{2,3)}$ auf rein nervöse Innervationsvariationen des Sympathikus-den Wegfall der sympathischen Hemmung, der durch die Verletzung des Peritoneums bedingt istzurück. Shima ${ }^{8}$ beobachtete Adrenalinmydriasis bei einer Katze nach Exstirpation des Frontallappens oder Durchschneidung der oberen Hälfte des Rückenmarks und erklärte dies als Wegfall des Hemmungsmechanismus sympathischer Natur.

Für uns bleibt die Frage nach der Grundlage von unsrer Adrenalinempfindlichkeit der Pupille bei chronischer Nephritis noch eine offene. Über die Veränderungen der Nebenniere und die Vermehrung von Adrenalin im Blute von chronischen Nephritikern wurde schon mehrfach diskutiert. Aber die Veränderungen der Nebenniere (Hyperämie und Hyperplasie des Marks und der Rinde), welche besonders von französischen Autoren (Vaquet ${ }^{9}$, Aubertin und Ambard ${ }^{10)}$, Dopter und Gourand ${ }^{11)}$ u.a.) betont wurden, sind bei der Mehrzahl chronischer Nephritiker nicht nachgewiesen und kommen auch bei anderen Krankheiten vor, wo die Adrewaliumydriasis negativ ausfällt (Wiesel ${ }^{12)}$, Aschoff ${ }^{13)}$, Bittorf ${ }^{14}$, Goldschmidt ${ }^{15}$ u.s.w.). Ferner kam nicht nur der Nachweis der Ehrmann'schen Reaktion mit dem Blutserum vou chronischen Nephritikern, was Schur und Wiese ${ }^{16) 1 ;}$ behaupten, zu keinem übereinstimmenden Resultat, sondern die Zunahme von Adrenalin oder 
adrenalinähulicher Substanz ist auch bei derselben Krankheit auf biologichem Wege noch unbewiesen (Fraenkel ${ }^{15}$ ) Janeway und Park ${ }^{19)}$, Kretschmer ${ }^{20)}$ u.a.). Wir ${ }^{21)}$ haben mittelst der Trendelenburgschen Methode ebenfalls negative Ergebnisse bekommen.

Hinsichtlich der Frage, ob das künstlich in den Kreislauf übergefülırte Adrenalin einen p sitiven Ausfall der Adrenalinmydriasis hervorruft, beziehungsweise begünstigt, haben wir bei gesunden Menschen und auch bei akut nephritischen Kranken mit negativer Pupillenreaktion 10 bis 15 Minuten nach der Adrenalininstillation 1 ccm Adrenalin subkutan injiziert, konnten aber keine Erweiterung der Pupille an der eingeträufelten Seite erzielen. Ferner konnten wir durch diese Behandlung die Instillationsmydriasis bei chronischen Nephritikern nicht verstärken.

Anf Grund der oben aufgestellten Tatsachen ist es nicht berechtigt, den Mechanismus der Adrenalinmydriasis bei chronischer Nephritis in der Vermehrung ron Adrenalin oder adrenalinähnlicher, die Sympathikusendigung direkt reizender Substanz zu erblicken. Mit grosser Wahrscheinlichkeit ist dies auf die von $u^{21)}$ nachgewiesene Substanz im Serum von chronischer Nephritis, die die Erregbarkeit dus Sympathilus steigert, zu beziehen.

\section{Zusammenfassung.}

Bei chronischer Nephritis resp. Schrumpfniere kommt Mydriasis durch Adrenalininstillation zustande. Dieses Phänomen geht meistens parallel mit der Höhe des Blutdrucks. Bei akuter Nephritis fällt es nur ganz selten positiv aus.

\section{Literatur.}

1) Loewi, Über eine neue Funktion d. Pankreas etc. Arch. f. exp. Path. a. Pharm. Bd. 59, 1908, s. 91.

2) Zak, Zur Kemtnis d. Adrenalinmydriasis. Verh. d. Kong. f. inn. Med. Bd. 25,1908, s. 398 .

3) Zak, Exp. u. klin. Beobachtung ä. Störungen sympath. Innervationen (Adrenalinnydriasis) u. ü. intestinale Glykosurie. Pflüger's Arch. Bd. 132, 1910, S. 147.

4) Cords, Die Adrenalinmydriasis u. ihre diagnostische Bedeutung. Wiesbaden 1911.

5) Raziejewski, Über d. augenblicklichen Stand der Kenntnis v. d. Nebennieren 11. ihren Funktionen. Berl. klin. Woch. 1898, Nr. 26.

6) Boruttau, Erfahrungen ü. d. Nebennieren. Pflüger's Arch. Bd. 78, 1899, S. 112 . 
7) Eppinger, Falta u. Rudinger, Über d. Wechselwirkungen d. Trüsen innerer Sekretion. Zeitschr. f. klin. Med. Bd. 166, 1908, S. 1.

8) Shima, Über d. Erweiterung d. Pupille bei Adrenalineinträufelung u. ihre Abhängigkeit roni Zentralnervensystem. Pflüger's Arch. Bd. 126 u. 127, 1909.

9) Vaquez, Hypertension arterielle. Arch. génér. de Méd. 1904.

10) Aubertin et Ambard, Lésion des surrénales dans les néphrites avec hypertension. Arch. génér. de Med. 1904.

11) Dopter et Gourand, Les capsules surrénales dans urémie expérimentale. Comptes rendus de la Soc. de Biol. Bd. 56, 1904.

12) Wiesel, Renale Herzhypertrophie u. chromaffines System. Wien. klin. Woch. 1907, Nr. 14.

13) Asch off, Bemerkungen zu der Schur-Wiesel'schen Lehre $\%$ d. Hypertrophie d. Nebennierenmarks bei chronischen Erkrankungen d. Nieren u. d. Gefässapparate. XII. Tagung d. Deut. pathol. Gesellschaft. 1908.

14) Bittorf Die Pathologie d. Nebennieren u. d. Morb. Addisonii. Jena 1908.

15) Goldschmidt, Beiträge z. Kenntnis d. Pathologie d. menschl. Nebenniere. Deutsche Arch. f. klin. Med. Bd. 98, 1909.

16) Schur u. Wiesel, Die Wirkung d. Blutserums v. Nephritikern auf d. Froschauge. Wien. klin. Woch. 1907, Nr. 23.

17) Dieselben, Üher d. chemischen Nachweis $\nabla$. Adrenalin im Blute v. Nephritikern. Wien. klin. Woch. 1907, Nr. 27.

18) Fraenkel, A., Uber d. Gehalt d. Blutes an Adrenalin bei chronischer Nephritis u. Morbus Basedowii. Arch. f. exp. Path. u. Pharm. Bd. 60, 1909, S. 395.

19) Janeway u. $\mathbf{P a r k}$, The question of epinephrin in the circulation and its relation to blood pressure. Journ. of exp. Med. Bd. 16, 1912, S. 541.

20) Kretschmer, Über d. Ätiologie d. nephritischen Blutdrucksteigerung $u$. verschiedene Untersuchungen $\ddot{u}$. $d$. Blutdruck steigernde Substanzen. Verh. d. Kongresses f. inn. Med. 1910, S. 731.

21) Kato u. Watanabe, Über d. Wirkung d. Serums $\nabla$. chronischen Nephritikern auf d. sympathischen Nerven. Tohoku Journ. of exp. Med. Bd. 1, 1920, S. 167 . 